\title{
Influential Factors of Geo-Information Sharing
}

\author{
Shanti Basnet \\ Survery Officer \\ Survey Department \\ Kathmandu, Nepal \\ peacebsnt@gmail.com; basnet26050@itc.nl
}

\begin{abstract}
Information resources, spatial or non-spatial are used widely and wisely to improve the organization's operation in today's competitive environment. With the advancement of information and communication technology, information sharing is made feasible and practiced within various entities. However, information sharing can be complex. There are various factors that influence cross-boundary information sharing because each organization operates within complex information, organizational and national context. There can be differences in technology, knowledge, culture, politics, geography, resources, relationships and intentions. This paper highlights on the different factors that can influence the information sharing in different perspectives.
\end{abstract}

\section{Keywords}

Geo-information, sharing, cross-boundary, influential factors

\section{Introduction}

Information is a key resource in today's organizational environment. As stated by (Kolekofski, Heminger, 2003), Richard Nolan, in his article, "Computer Data Bases: The Future is Now" was one of the first to study the importance of information as an organizational resource. Though being crucial, it is often not shared and used. It has not been utilized to the extent as it could be. Information is associated with the objective in order to benefit the organization which also includes the sharing of information amongst those who can make profitable use of it.

When information is held by different stakeholders, exchange of information and materials is a must for resource combination (Haeussler, 2011). The information shared allows building on each other's work and achieve the result in faster and economical manner. Sharing information is considered as an important approach to increase organizational efficiency and performance (Steinel, Utz, Koning 2010). It has been made feasible due to the advances in information and communication technology. However, sharing information is a complex task (Steinel, et al 2010). The scientific progress and its societal benefits also hinge on the sharing of the information (Hauessler, Jiang, Thursby). New information plus the advancement in sciences is also created mainly through two generic processes: exchange and combination (Nahapiet, Ghosal).

Sharing of the information is found to be done in two major forms: one- on-one and the General Sharing. General Sharing takes place with the scientific community as a whole for example, through conference presentation (Hauessler, Jiang, Thursby). The one -on- one Sharing requests mostly concerned research- related information or research input that is, information which is often not published or cannot be published in journal, but provides the basis for research findings, for example software, database, description of laboratory process.

There are different perspectives and aspects on which the sharing of the information is affected.

\section{Information Sharing in Different Perspective}

Since few years, organizations have shifted from information protection approach to information sharing as a new goal (Steinel, et al 2010). Though sharing of information has received much attention, initiatives has often failed due to various reasons. Hence, to facilitate the cross boundary information sharing, it is critical to establish an understanding 
of factors that influence sharing and maintain collaborative relationships (Yang, et al 2012).

Moreover, researches have revealed that information sharing involve complex interactions between the participants due to the differences in their origins, values and the cultures [Gil-Garcia, 2010; Lam, 2005; Yang, et al 2012]. Within the national context, information sharing can be performed in three different perspectives (Slaughter): Interpersonal Information Sharing, Intra- organizational Information Sharing and Inter- organizational Information Sharing.

\subsection{Interpersonal information Sharing}

Interpersonal relationship is developed within many contexts: colleagues, neighbors, classmates, or members of community (Yang, et al 2011). Sharing information at the interpersonal level focuses mainly on the behavior that each individual have such a motivations, approaches and channels for an individual to share information with others. Information can be shared voluntarily in order to provide information to the people who are in need (Jarvenpaa, Staples; 2001). The information is shared among the needy using the simplest and cheapest technology available such as email and face-to-face (Erdelez, Rioux, 2000). The main reasons for sharing the information in these levels are (Marshal, Bly, 2004):

a. To establish mutual awareness between the information giver and taker

b. To educate or raise consciousness

c. To develop rapport

The information shared here is to strengthen social ties and relationships between the provider and recipient and reflect the common interest between them. However, information sharing in individual interest may also interact with various organizational factors like competition and collaboration that tend to hinder information sharing behavior.

Sharing of the information is also found to be influenced by the individual's belief and attitude about the information and its sharing. Beliefs influence the overall attitude about any object and beliefs, attitude, intentions and behaviors are interlinked (Kolekofski, Heminger, 2003). An experimental finding has shown the perception of organizational ownership and attitudes for information sharing differs with the type of information. Individuals are more liable to share personal knowledge that the tangible knowledge. Interpersonal relationship also plays an important role for sharing the information. (Kolekofski, Heminger, 2003) As suggested, "Belief about the information, interpersonal relationships, organizational factors and task relevancy determine an ownership or stewardship attitude which subsequently influence the likelihood of sharing information"

\subsection{Intra- organizational Information Sharing}

There is trend to encourage groups to share the information that they have within organizations (Dawes, Gharawi, Burke, 2012). Though in the bureaucratic model, the flow of information is strictly controlled. Due to this limited access of information and its sharing, one lacks the capability to develop integrated solutions of the problems. Literatures have revealed that there are many influencing factors for intra- organizational information sharing. The relationships between the factors are complex and have influence on each other. The following figure 1 shows the relationships between the factors which are present in three layers.

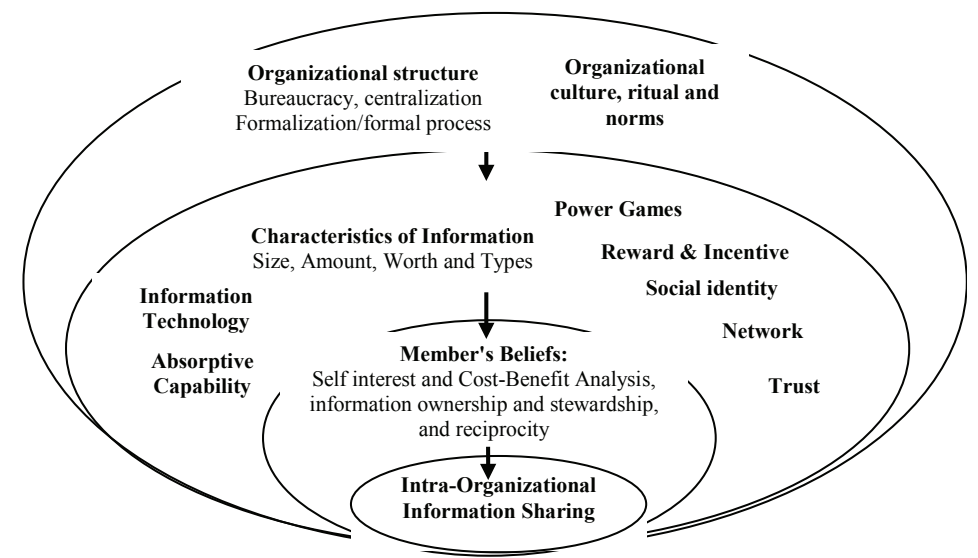

Figure 1: Factors influencing intra-organizational information sharing [16] 
Layer 1: Though the ideal bureaucracy is efficient and fair organization, if it gets bigger the distributed duties in different hierarchies and sub units become reason of decreased efficiency. It may bring obstacles to share information between different departments because of having different functional mandates (Yang, et al 2011). Similarly the attitude and actions of organizational members for information sharing are highly influenced by organizational cultures, values and norms. When the culture of the organization makes emphasis on the fairness, affiliation and innovation, it can positively influence the intentions to share information. Information sharing may clash with the culture of the organization if its value is not a part of the organization's culture (Zheng et al, 2005).

Layer 2: Researchers emphasize on the importance of the incentive system to motivate the members of organization to share the information within different groups and departments (Yang, et al 2011). Performance based reward system is more likely to sharing of knowledge and information within members of organization and so is the bonus system which may also increase the quality of the information shared (Kolekofski, Heminger, 2003). Owning information is considered as owning power within an organization. Therefore information is taken as an asset and used by organization members to elevate their power and sharing it is viewed as loss of individual power and social influence within organization. Hence, sharing of information is less if more power game exists. Social networks as well are important to promote the information sharing. It includes individual and group contacts, communications and interactions that can promote trust and relationships that can enhance the sharing behavior (Kolekofski, Heminger, 2003). Some people always questions that why individuals require the information they possess while others will always keep an open mind to the requests for information in their possession. Trust is a critical factor between the involving individuals to share the knowledge and information (Zheng et al, 2005) and is willing to share if they feel that they are protected against opportunistic people. Moreover, the lacks of trust amongst organizational members create obstacles in sharing information. (Kolekofski, Heminger, 2003) The size, amount, type and perceived value for the requested information also influence the attitudes and intentions of the members of the organization to share the information. Two types of knowledge explicit and tacit are owned; explicit knowledge is objective and rational those are expressed in words, numbers, formulas and charts while tacit knowledge is subjective, difficult to communicate and express and based on experiences (Yang, et al 2011). Absorptive capacity depends on the prior knowledge, ability to recognize the value of new information and to assimilate and apply it on the practical and innovative use. Having this capacity individuals within organization are better capable to receive and use the shared information. The experienced has proved that the advancement of information technology develops information system to facilitate sharing of the information. However, if the implemented information technology is not easy and used efficiently, individuals within an organization, the use of IT will be lower and activities to share will tend to be lesser and sometimes could be negatively influenced (Yang, et al 2011).

Layer 3: Self interest of individual can reduce support for information sharing within an organization. "The information of any organization that is represented as a product is easier to share between individuals and is considered as property owned by the organization while information such as expertise is more difficult to share and considered as individual property" (Yang, et al 2011). Reciprocity is another force to drive the behavior of information sharing. The anticipated reciprocity is an important factor to positively influence the attitudes towards the sharing the information.

\subsection{Inter-organizational Information Sharing}

Some of the factors discussed above may also be applied to the inter-organizational scenarios. The public sectors have increasingly realized the importance of sharing information to improve the efficiency of the government agencies (Gil-Garcia et al, 2005). Interoperability across organizations represents cross boundary information sharing (Landsbergen, Wolken, 2001). The most influential factors viewed and defined by (Dawes et al, 2012; Zheng et al, 2009) are in three primary perspectives: Technology, Management and Policy. These three are the core elements in inter- organizational information sharing. The relationship between these three has been shown in the given figure 2 . 
The technological perspective: It is experienced that different organization have various types of hardware and software and it is a challenge to integrate heterogeneous information system in varying platforms, data quality, standard and schemas. Another critical factor for the cross- boundary information sharing is the levels of technological capability of various organizations. However, the advancement in the information technology has enhanced the effectiveness and efficiency of the inter - organizational collaboration (Zheng et al, 2005). Different frameworks are being applied to bride such information systems and heterogeneous databases with inconsistent data structures and definitions. The adoption of IT can also be a challenge because of the security and confidentiality, it is critical to design a system that can handle access authorization and authentication for shared information.
The organizational perspective: Researcher has specified that information sharing can involve complex interaction between participating organization due to the differences in their origins, values and culture. It is also pointed that some governmental organization with little or no knowledge of sharing information lacks the basic understanding of the benefits that can occur from cross boundary information. In addition to this, due to the bureaucratic organizational boundaries, government organizations are less aware of what information can be shared and retrieved from other agencies. Some other factors that resist the inter - organizational information sharing are traditional business process, perception of losing benefits and lack of resources. Organizations are not willing to share, without appropriate compensation, also because they have spent lots of budget, time, staffs and other resources to build the knowledge and information

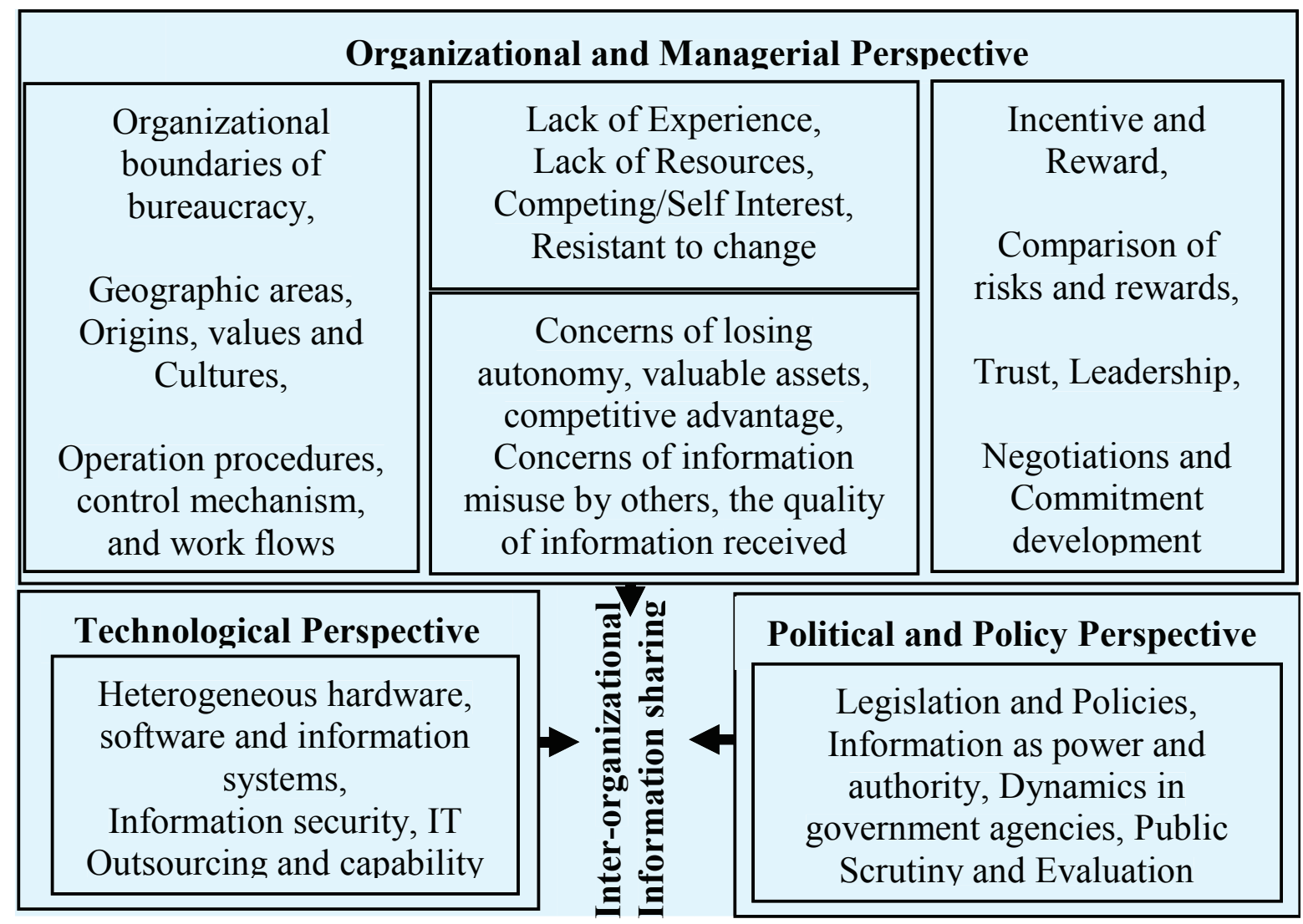

Figure 2: Factors influencing inter-organizational information sharing in public sector 
The political and policy perspective: Legislation and policy influence strongly to share information and across organization especially in the public sector (Gil-Garcia et al, 2010). Researchers have found that legal and policy regulations can facilitate relationship building, risk reduction and trust development in inter-organizational information sharing when specific guidance on how to utilize information is proposed. Without the support of legislatures and policy makers, inter-organizational information sharing in public sector can be lose its priority status and lack necessary funding and resources to make projects sustainable (Zheng et al, 2005). However, laws and regulations may also sometimes be a barrier to obstruct cross-boundary information sharing in public agencies. Sharing of the information can be hindered because of policies itself that prohibit the information sharing of sensitive and regulated information for maintaining public safety and national security.

\section{The Boundaries of Information Sharing}

The discontinuities that represent the incoherence and gaps between the entities in the context of information sharing in different environments, tasks and relations to others are considered as boundaries (Yang et al, 2012). The difficulty of crossing the boundary may be lower if there exist no significant boundaries or has been removed. Barriers hence can be eliminated or overcome by using some efforts even if it tend to exist for a longer period of time and significant institutional changes has been made. Hence organizational boundary in information sharing and integration is also observed in two different dimensions: vertical and horizontal (Yang et al, 2012; 19). Organizational, personal, sectoral, geographic, development level and process boundaries falls within the vertical and horizontal dimensions. Researchers have claimed that boundaries in both the dimensions: vertical and horizontal are equally important and may exist simultaneously.

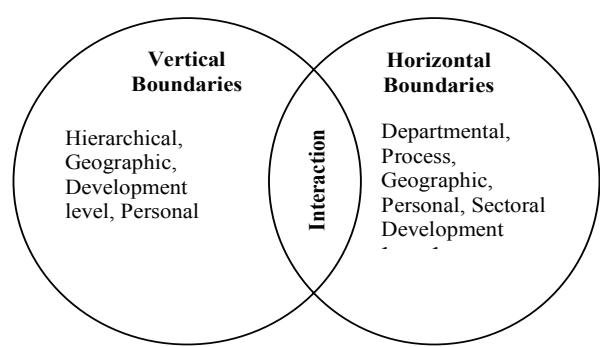

Figure 3: An integrated framework of boundaries in information sharing and integration (Yang et al, 2012)

\section{The Geo-Information Sharing}

Similar to other information, the speedy development and increased demand of the geographic information infrastructure has made geographic information an important tool in policy planning and decision making. Global issues, such as climate change, food and energy crises, peace operations and humanitarian assistance, all necessitate strong support for geographic information management on a global scale. Moreover, in the digital era location or place, is gaining increasing importance making the efficient and effective management of information a priority in both the public and private sectors and even more at the personal level. Over eighty percent of existing digital databases have a locational component. "Everything is somewhere" and location can be used as an integrator for both quantitative and qualitative information (Taylor, 2010). Additionally society is increasingly using location based information in day to day life including GPS, Google Earth and related spatially referenced databases knowingly or unknowingly. Governments too are using location or place in planning and budgeting.

Different organizations have quite different legal and policy mandates and these differences constrain the effective cooperation and sharing of the information between them. As in the case of other non spatial information, there are many barriers to the sharing of geo data and information too, which include technical, legal, administrative and political barriers. The technical barriers include the issue of interoperability. Although the technical issues are significant they are perhaps much easier to resolve than the legal, administrative and political ones (Taylor, 2010). Though it is widely recognized that collecting data multiple times for the same purpose is wasteful and inefficient, yet it continues to occur. Inadequate amount of spatial data is available and they too are limited by the quality, accuracy, and completeness of the underlying geospatial data. Constraint exists also on the knowledge of what data can be shared and in what form. Duplication of information exists and there is necessity to update several sites. The major difficulties faced in this domain are the multiplicity of spatial representations, the discrepancy regarding coordinates and some administrative constraints. (Laurini, 1994). To overcome these drawbacks standardization and sharing geo-information can be very fruitful. 
"Technical interoperability has provided geographic information communities with substantial improvements for constructing geographic information system (GIS) capable of very low friction and dynamic data exchanges. These technical advances stand to provide substantial advantages for sharing geographic information however reaping these advantages in highly heterogeneous operational and organizational environments requires the understanding and resolution of semantic differences" (Harvey, Kuhn, Pundt, Bishr, Riedemann, 1999). Though different efforts have been made in this regard, obstacle still remains pertinent across organizations for data sharing and future developments of standards. Although the goals of transparent data exchange and remote access have to be reached by technical interoperability, work on interoperability provides a basis for facilitating data sharing and helping resolve redundancy problems (McKee 1998).

Ideally, spatial data/information sharing efforts would produce a national spatial data infrastructure, (NSDI). In addition to promoting the efficiency and interoperability of such a national system, NSDI is often promoted as "digital infrastructure" on par with other parts of the nation's critical infrastructure and underscore its role in the national economy and in national security. Cooperation is necessary to realize the overall vision of the NSDI, which is to assure that spatial data from multiple sources are available and easily integrated to enhance the understanding of our physical and cultural world (Folger, 2010).

\section{Conclusion}

Whether it is spatial or non-spatial information, it's sharing can neither be supervised nor imposed. It is influenced by many factors related to the environment and domestic relationships of the people and organization. The contextual difference among them generates divergence in the culture, intention, politics, knowledge, resources, geography, technology and varying organizational factors. However, if the individuals and organizations follow the norm of open science with the stronger conviction, there will be maximum probability for the information sharing. It is necessary to strengthen the norms and norm based mechanism which helps stakeholders to share information for the promotion of knowledge.

\section{References}

Dawes, S.S., Gharawi, M.A., Burke, G.B. (2012): Transnational Public Sector Knowledge Network: Knowledge and Information Sharing in a Multidimensional Context. Government Information Quarterly. 29

Erdelez, S.\& Rioux, K. (2000): Sharing Information Encountered for Others on the Web. New Review of Information Behavior Research. 1,219-233

Folger, P. (2010): Geospatial Information and Geographic Information System: Current Issues and Future Challenges. Congressional Research Service, Report for Congress.

Gil-Garcia, J.R. \& Pardo, T.A. (2005): E-Government Success Factor: Mapping Practical Tools to Theoretical Foundations. Government Information Quarterly. 22187-216

Gil-Garcia, J.R., Pardo, T.A.\& Burke, G.B. (2010): Conceptualizing Information Integration in Government. In Scholl, J. Electronic government: Information, Technology and Transformation, 179202

Harvey, f., Kuhn, W., Pundt, H., Bishr, Y., Riedemann, C. (1999): Semantics Interoperability: A Central Issue for Sharing Geographic Information. The annals of Regional Science 33:213-232

Haeussler, C., Jiang, L., Thursby, J., Thursby, M.: Specific and General Information Sharing. NBER Working Paper 15315

Haeussler, C. (2011): Information-Sharing in Academia and the Industry: A Comparative Study. Research Policy. 40, 105-122

Jarvenpaa, S.L.\& Staples, D.S. (2001): Exploring Perceptions of Organizational Ownership of Information and Expertise. Journal of Management Information System. 18 151-183

Lam, W. (2005): Barriers to e-Government Integration. Journal of Enterprise Information management. 18, 511-530

Landsbergen, D. J.\& Wolken, G. J. (2001): Realizing the Promise: Government Information Systems and the Fourth Generation of Information Technology. Public Administration Review. 6, 206-220 
Laurini, R. (1994): Sharing Geographic Information in Distributed Databases. URISA p441-454

Marshal, C.C.\& Bly, S. (2004): Sharing Encountered Information: Digital Libraries Get a Social Life. Paper presented at the International Conference of Digital Libraries, Tuscon, AZ

Nahapiet, J. \& Ghosal, S.: Social Capital, Intellectual Capital and the Organization Advantage. Academy of Management Review 23 (2) 242-266

Slaughter, A.: A New World Order. Princeton, New Jersey: Princeton University Press

Steinel, W., Utz, S., Koning, L. (2010): The Good, the Bad and the Ugly thing to do when sharing Information: Revealing, Concealing and Lying Depend on Social Motivation, Distribution and Importance of Information. Organizational Behavior and human Decision Processes.113, 85-96

Taylor, D.R.F (2010): Global Geographic Information Management: Some Institutional and Data Sharing Issues in Integrating Geospatial and Statistical Data, Presentation to the Second Preparatory Meeting of the Proposed United Nations
Committee on GlobalGeographic Information Management, Newyork

Yang, T.M. \& Maxwell, T.A. (2011): InformationSharing in Public Organizations: A Literature Review of Interpersonal, Intra-personal and Interorganizational Success Factors. Government Information Quarterly. 28, 164-175

Yang, T.M., Zheng, L., Pardo, T. (2012): The Boundaries of Information Sharing and Integration: A Case Study of Taiwan Government. Government Information Quarterly. 29, 551-560

Zheng, J., Dawes, S. S., \& Sarkis, J. (2005). Exploring Stakeholders' Expectations of the Benefits and Barriers of e-Government Knowledge Sharing. The Journal of Enterprise Information Management, 108(5), 548-567

Zheng, L., Yang, T.M., Pardo, T.A. \& Jiang, Y. (2009): Understanding the "Boundary" in Information Sharing and Integrating. Proceedings of the $42^{\text {nd }}$ Hawaii International Conferen

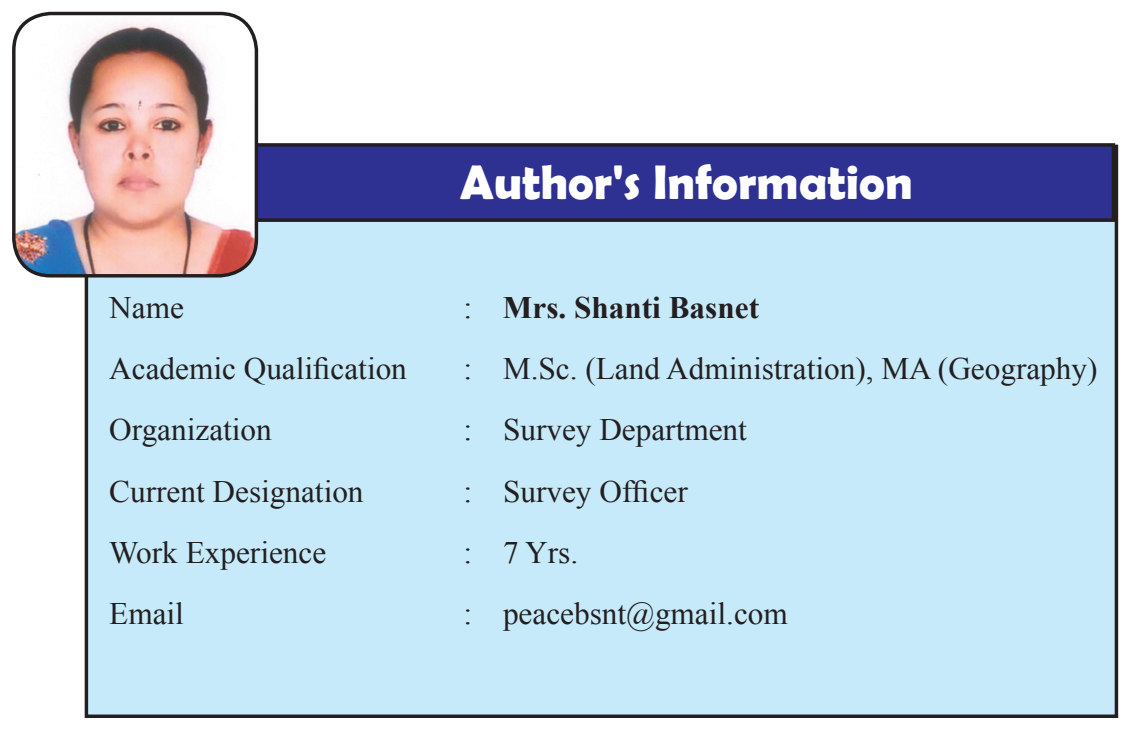

